\title{
Moving Object Segmentation in a Video Sequence using Optical Flow and Motion Histogram Technique
}

\author{
Mehul C Parikh \\ Phd Scholar \\ Charotar University of Science and Technology \\ Changa, Gujarat
}

\author{
Kishor G. Maradia, Ph.D \\ Associated Professor (E.C) \\ Government Engineering College \\ Modasa
}

\begin{abstract}
The moving object detection and tracking in video sequence is significant research area in the field of computer vision and image processing. Object detection and tracking is key step for object recognition, navigation systems and surveillance systems. An effort has been made to develop an approach for moving object detection in video sequence, based on motion segmentation using optical flow and motion histogram. The algorithm has been described in detail and its competence was checked by performing simulation experiments. ROC comparison shows that this algorithm gives better result than traditional methods.
\end{abstract}

\section{Keywords:}

Motion Segmentation, Feature Extraction, Object Detection, Optical Flow, Motion Histogram, Receiver Operating Characteristic Curve

\section{INTRODUCTION}

Intellectual video surveillance techniques is necessary for security purpose, abnormal behavior detection, traffic system, etc. Critical task in this surveillance system is moving object detection from the video sequence. This task is difficult because there are multiple foreground objects, texture of objects are different, intensity changes are abnormal, vibrant background, occlusion, shadow effect etc. Motion can be detected by numerous techniques like frame difference, region base, clustering, background subtraction, histogram, edge detection methods. Labeling to pixel based on motion is used for motion detection. The difficult task in video sequence is to track object in consecutive frames as it is to establish relation between objects and it's parts. Frame difference and background subtraction method are based on illumination changes which effects the intensity of pixel and these pixels are considered as foreground object. These methods have many limitations, one of them is that these methods are not able to distinguish between vibrant background and foreground. Histogram techniques is also grouped pixel of various intensity, this information is useful for labeling pixel. Optical flow uses intensity changes of pixel and magnitude to generate direction of motion vector which is an important indication for detecting moving object in video sequence. To overcome vibrant background detection problem and accurate detection of foreground, we present method which uses optical flow and motion histogram in combination to remove vibrant background and detect foreground accurately. Finally morphological operation was used to get true shape of the object.

The overall structure of the paper is organized as follows : Section II presents a literature review in object segmentation, moving object detection. Section III provides detail of proposed algorithm. Section IV shows experimental results and discussion Section V gives quantitative evaluations and computational cost analysis and conclusion in section VI.

\section{LITERATURE REVIEW}

Following section summarizes several mathematical models for detecting moving object in video sequence. In frame difference method, difference of pixels of two consecutive frame is taken, if the difference is small the object is considered as static, otherwise it is moving. In background subtraction algorithm, background is subtracted from foreground and value of every pixel compared with threshold. If pixel value is higher than the given threshold, the pixel point is foreground, other wise it is background. The algorithm is simple can work precisely under unchangeable background and can detect moving object exactly. But the major limitation is that it is very sensitive to illumination changes. Soon-Yong Park et al.[1] suggested the method to track corner feature of the background frame by frame. Method register background image obtained by transformation, then extraction of the foreground object was done by combing object correlation and accumulation of frame difference image. Jie Cao, Li Li [3] suggested method to detect moving vehicle by first initialization of background. The first frame in $(t=0)$ was assumed as the background, after that inter-frame difference was calculated, fast median filter was used for filtering the difference image to segment moving vehicle. Liu Liu et al.[4] suggested algorithm based on motion edge feature, which was based on gray histogram and canny operator. Jie $\mathrm{He}$ et.al [5] proposed algorithm to detect moving object based on edge characteristics and inter frame difference. Shijie Zhang et al. [6] proposed an attention based region growing algorithm. Saeed Kamel et al. [7] proposed a novel approach to separate moving objects from back ground based on combination of kalman filtering and background subtraction. Adaptive motion histogram technique was proposed by Wei Zhang et al.[8]. B.D. Lucas and T. Kanade[9] suggested optical flow approach based on two consecutive images with time difference $t$. In computer vision, this method is widely used for optical flow estimation. It assumes that the flow is essentially constant in a local neighborhood of the pixel under consideration, and solves the ba- 
sic optical flow equations for all the pixels in that neighborhood, by the least squares criterion. By combining information from several nearby pixels, the Lucas-Kanade method can often resolve the inherent ambiguity of the optical flow equation. It is also less sensitive to image noise than point-wise methods.Anand Paul et al.[13] proposed block based motion estimation algorithm, in which each block was determined based on motion vector and prediction error obtained from the previous block. Algorithm reduce the computational complexity of motion estimation. All these methods were designed and tested for a particular situation and their results shows that methods were successful for test situation only. Also these methods lack time efficiency while some lack robustness.

\section{PROPOSED ALGORITHM}

For developing a robust method which should eliminate all the shortcomings existing in previous motion segmentation techniques such as tree weaving and change of intensity in the dynamic background, we here by propose an algorithm for motion segmentation based on "Optical Flow field and Motion Histogram". Figure 1 shows the block diagram of the proposed algorithm.

\subsection{RGB to Gray scale conversion}

The algorithm is working upon the two successive frames of a video sequence. As optical flow method is based on the intensity of the pixels in the successive frames, the RGB to gray scale conversion of the image is mandatory. The RGB image is transformed to 8 bit quantized gray level image. All of the subsequent frames should be converted from RGB to gray scale as video sequence moves on.

\subsection{Optical Flow}

Optical flow reflects the image changes due to motion during a time interval $d_{t}$, and the optical field is the velocity field that represents the three-dimensional motion of object points across a twodimensional image. Optical flow is an approximation of the local image motion based upon local derivatives in a given sequence of images. That is, in 2D it specifies how much each image pixel moves between adjacent images, while in 3D it specifies how much each pixel moves between adjacent volumes. In case of 2D, the moving patterns cause temporal varieties of the image brightness. It is assumed that all temporal intensity changes are due to motion only. In normal condition this assumption is true but many exceptional cases are there. Low pass and high pass filters are used for computation of 2D/3D derivatives [12, 14]. Hence following two step procedure were used for the computation of differential optical flow :

(1) Measure the spatio-temporal intensity derivatives and

(2) Integrate normal velocities into full velocities, [9, 11].

These algorithms developed for the image in which there can be no rigid object in scene, no occlusion, no expansion, no contraction or deformation. Image must be have real motion without above condition. With this type of image optical flow capture real motion in a scene.[14] The Lucas-Kanade method[9] assumes that the displacement of the image contents between two nearby instants (frames) is small and approximately constant within a neighborhood of the point $p$ under consideration. Thus the optical flow equation can be assumed to hold for all pixels within a window centered at $p$. Namely, the local image flow (velocity) vector $\left(\nu_{x}, \nu_{y}\right)$ must satisfy

$$
\begin{aligned}
& I_{x}\left(q_{1}\right) \nu_{x}+I_{y}\left(q_{1}\right) \nu_{y}=-I_{t}\left(q_{1}\right) \\
& I_{x}\left(q_{2}\right) \nu_{x}+I_{y}\left(q_{2}\right) \nu_{y}=-I_{t}\left(q_{2}\right) \\
& \cdot \\
& I_{x}\left(q_{n}\right) \nu_{x}+I_{y}\left(q_{n}\right) \nu_{y}=-I_{t}\left(q_{n}\right)
\end{aligned}
$$

where $q_{1}, q_{2} \ldots q_{n}$ are the pixels inside the window, and $I_{x}\left(q_{i}\right), I_{y}\left(q_{i}\right), I_{t}\left(q_{i}\right)$ are the partial derivatives of the image $I$ with respect to position $\mathrm{x}, \mathrm{y}$ and time $\mathrm{t}$, evaluated at the point $q_{i}$ and at the current time. This system has more equations than unknowns and thus it is usually over-determined. This method obtains a compromise solution by the least squares principle. These equations can be written in matrix form $\mathbf{A} \nu=\mathbf{b}$, where,

$$
\mathbf{A}=\left[\begin{array}{cc}
I_{x}\left(q_{1}\right) & I_{y}\left(q_{1}\right) \\
I_{x}\left(q_{2}\right) & I_{y}\left(q_{2}\right) \\
\vdots & \vdots \\
\vdots & \vdots \\
I_{x}\left(q_{n}\right) & I_{y}\left(q_{n}\right)
\end{array}\right], \nu=\left[\begin{array}{c}
\nu_{x} \\
\nu_{y}
\end{array}\right], \mathbf{b}=\left[\begin{array}{c}
-I_{t}\left(q_{1}\right) \\
-I_{t}\left(q_{2}\right) \\
\vdots \\
\vdots \\
-I_{t}\left(q_{n}\right)
\end{array}\right]
$$

This system has more equations than unknowns and thus it is usually over determined. The Lucas-Kanade method [9] obtains a compromise solution by the least squares principle. Namely, it solves the $2 \times 2$ system $\mathrm{A}^{T} A \nu=\mathrm{A}^{T} \mathrm{~b}$ or $\nu=\left(\mathrm{A}^{T} \mathrm{~A}\right)^{-1} \mathrm{~A}^{T} \mathrm{~b}$ where $\mathrm{A}^{T}$ is the transpose of matrix $\mathrm{A}$.

\subsection{Motion Histogram}

Foreground moving object indications are dissimilar to other objects. These dissimilarities draws attention to visualize their motion property. Here this property is used to segment the foreground object. The optical flow algorithm depicted in the previous section provides motion vectors which is used as an input to generate motion histogram. Motion vector represents the motion information in simplified way. This section explore the association between the motion vectors of the foreground object and vibrant background. Clip of the "Highway traffic" sequence having 100 frame were selected to analyze the different property of the foreground object and the vibrant background(Figure 2). First step is to select vibrant background area and the foreground object area. The motion vectors in the two selected regions were figured and the motion histograms was obtained as shown in figure 2 It can be seen from figure 2 that the motion vectors of the foreground object grouped in a lesser area, while the motion vectors of the vibrant background are dispersive [8]. Analysis of the direction and the magnitude of the motion vectors were carried out.

\subsection{Parameter Selection : Thresholding}

Motion mask was computed according to the method describe by Wei Zhang et. al.[8], let $\nu_{i, j}=\left(x_{i, j}, y_{i, j}\right)$ be the motion vector of the pixel $(\mathrm{i}, \mathrm{j})$ of $n_{t h}$ frame and let the motion histogram of $n_{t h}$ frame be $\psi_{n}$. The motion mask $\left(\zeta_{n}\right)$ was computed as follows [8]:

$$
\zeta_{n}(i, j)= \begin{cases}1 & \text { if } \nu_{i, j} \neq 0 \\ 0 & \text { else }\end{cases}
$$




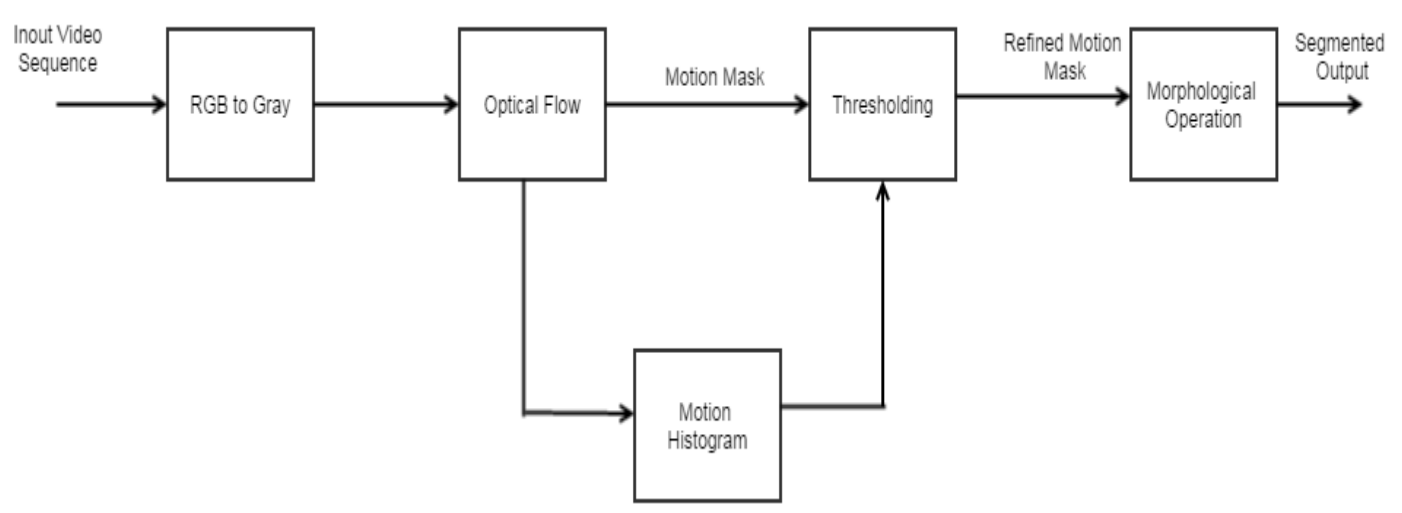

Fig. 1: Block diagram of optical flow approach.
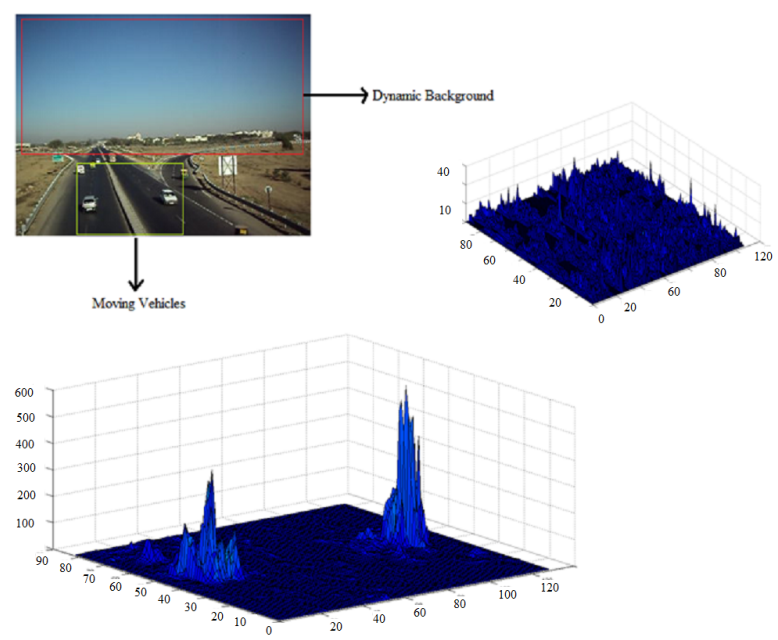

(a)

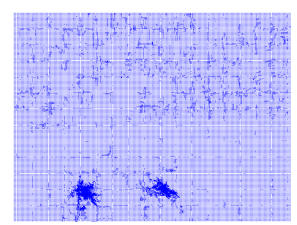

(b)

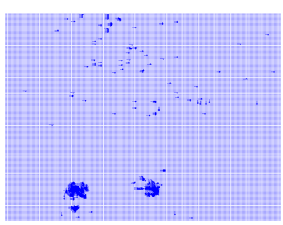

(c)

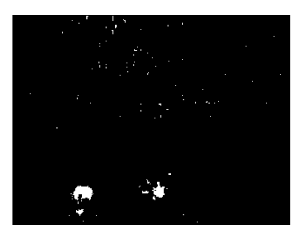

(d)

Fig. 2: (a)Highway test sequence with motion histogram of dynamic background and moving vehicles. (b) Motion vectors after optical flow. (c) Motion vectors after thresholding. (d) Refined motion mask.
The threshold $\theta$ was set to $\psi_{n}$ to get refined motion histogram $\left(\widetilde{\psi}_{n}\right)[8]$ :

$$
\widetilde{\psi}_{n}(i, j)= \begin{cases}\psi_{n}(i, j) & \text { if } \psi_{n}(i, j)>\theta \\ 0 & \text { else }\end{cases}
$$

The refined motion mask $\widetilde{\zeta}_{n}$ is defined as follows:

$$
\widetilde{\zeta_{n}}(i, j)= \begin{cases}1 & \text { if } \widetilde{\psi}_{n}\left(x_{i, j}, y_{i, j}\right) \neq 0 \\ 0 & \text { else }\end{cases}
$$

Figure 2 shows enormous regions of interferential moving objects caused by unstable background, while most of the interferential moving objects are removed. The refined motion mask can be produced by setting the threshold $\theta$ at maximum value of that of background motion vector, and thus we can get segmentation of only vehicles with lasser background discrepancy in figure 2 (c)). This indicates that the motion vectors can be used as the feature to detect the foreground object in the vibrant scene, which is similar to result of Wei Zhang et. al. [8]

\subsection{Morphological Opearion}

Obsrvation from figure (2/d)) indicates that the vehicles are not properly segmented owing to the aperture effect in calculating motion vectors of vehicles. Due to apreture effect some part of the vehicle can't be segmented and that part of the vehicle is considered as a background. We term this condition false negative when foreground is classified incorrectly as the background. In addition some of the large background movements produce higher values of motion vectors and some of them can't be removed after thresholding. Therefore some of the background part is segmented as a foreground and if background is classified incorrectly as the foreground we consider it as a false positive. The inadequacy produced by aperture effect and large background movements can be improved by applying mathematical morphology (MM) to the refined motion mask. Mathematical morphology (MM) is a theory and technique for the analysis and processing of geometrical structures, based on set theory, lattice theory, topology, and random functions. In binary morphology, an image is viewed as a subset of an euclidean space $R^{d}$ or the integer grid $Z_{d}$, for some dimension d. Morphological operation is executed upon refined motion mask as shown in figure (3). Initially closing operation with an open disk of radius $\mathrm{r}=1$ was performed to remove false positives. Closing operation was 
followed by dilation operation with a $5 \times 5$ square to add boundaries, removed due to opening operation. And finally closing operation is performed to remove false negatives. The results of morphological operation are illustrated in figure (3)

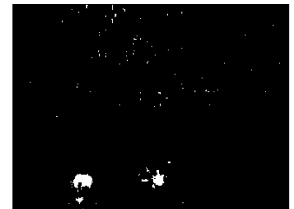

(a)

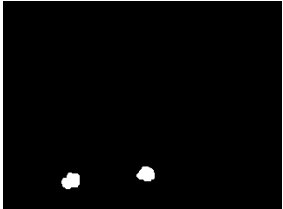

(b)
Fig. 3: (a)Result refined motion mask. (b) Result after morphological operation.

\section{EXPERIMENTAL RESULTS AND DISCUSSION}

We have verified the method on numerous traffic flow sequences to evaluate its usefulness. In this paper results of three different sequences are displayed. Similar results were obtained on other sequences. The proposed algorithm was implemented with MATLAB software on $2.6 \mathrm{GHz}$ PC with $2 \mathrm{~GB}$ RAM. First step is transformation of the RGB image into gray-scale image of 8 bit quantization of gray level. Three typical scenes are used in the experiments to evaluate the performance of the proposed method, "Black car sequence", "Multiple Car sequence", and "Highway traffic sequence". The result obtained were compared with the result of frame difference and background subtraction method.

\subsection{Single Black Car Sequence (Test Sequence 1)}

Figure 4 shows original sequence. Figure 5 represent its motion historgram. Result of optical flow algorithm as motion vectors followed by refined motion vectors as an outcome of thresholding function and finally refined motion masks are shown in Figure 6. Figure 7 displays result of frame difference, background subtraction and the result of proposed method and ground truth. In this sequence moving object is black car and due to less resolution on road some intensity changes are noted. Result of frame difference shows that the black colored moving car is moderately detected and back ground subtraction result shows bulky region as foreground object which is due to the boundary of the stationary objects. Result of our proposed method shows that the black colored foreground object(car) is segmented successfully and filtered all the small movement caused by the intensity changes. Figure 8 . shows result comparison on ROC Curve detail of which are discussed in next section.

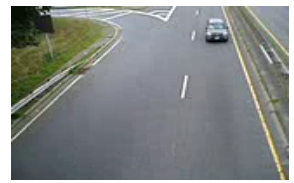

(a)

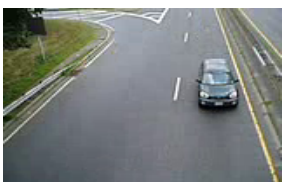

(b)
Fig. 4: Black car sequence.

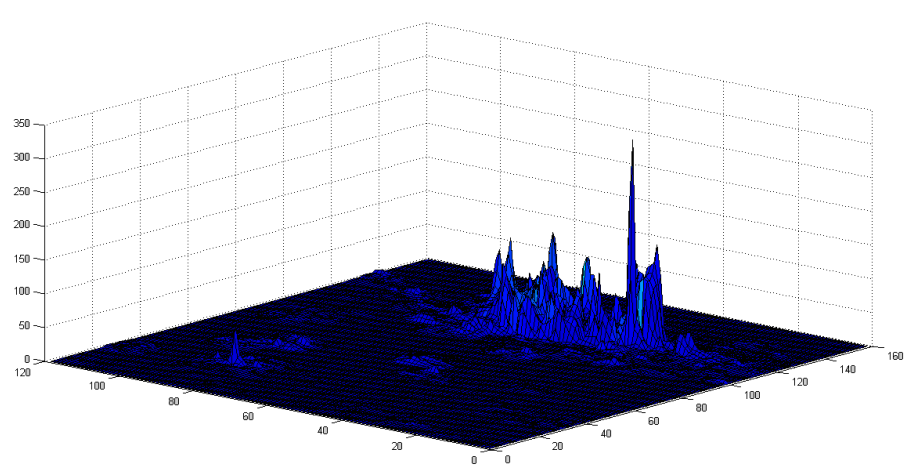

Fig. 5: 3 - D Motion histogram for black car sequence.

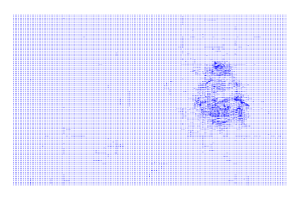

(a)

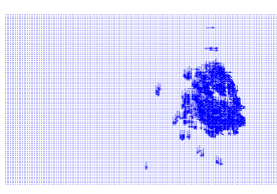

(b)

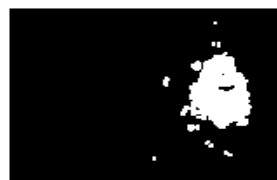

(c)

Fig. 6: (a) Motion vectors. (b) Refined motion vectors. (c) Refined motion mask.

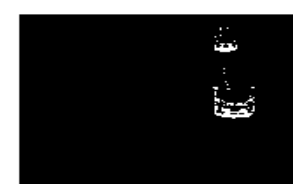

(a)

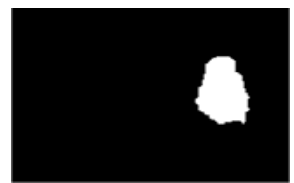

(c)

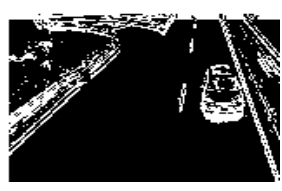

(b)

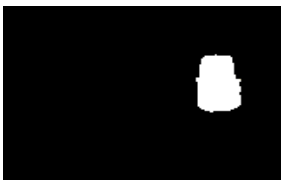

(d)
Fig. 7: (a) Result of frame difference method. (b) Result of background subtraction method. (c) Result of proposed method. (d) Ground truth. 


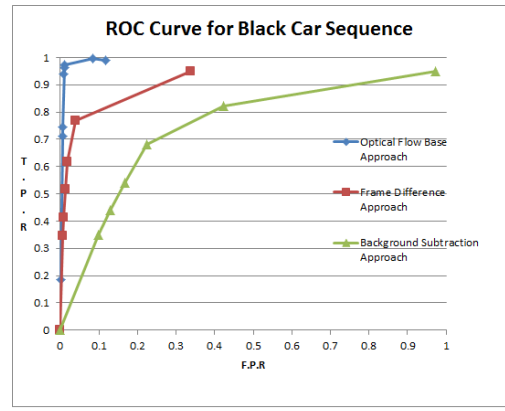

Fig. 8: ROC curve for black car sequence.

\subsection{Three Car Sequence (Test Sequence 2)}

Figure 9 shows original sequence. Figure 10 presents its motion historgram. Result of optical flow algorithm as motion vectors followed by refined motion vectors as an outcome of thresholding function and finally refined motion masks are represented in Figure 11. Figure 12 displays result of frame difference, background subtraction and the result of proposed method and ground truth. In this sequence moving object is black car and white car and due to less resolution on road some intensity changes are noted. Result of frame difference show that foreground objects are only moderately segmented and some part of background are also detected as a foreground, we term this condition as a false car detection and back ground subtraction result shows bulky region as foreground object which is due to the boundary of the stationary objects. Result of our proposed method shows that the black colored foreground object(car) is segmented successfully and filtered all the small movement caused by the intensity changes. Figure 13 . shows result comparison on ROC Curve.

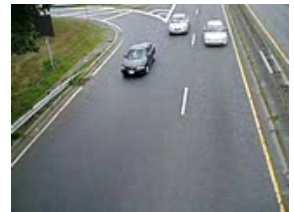

(a)

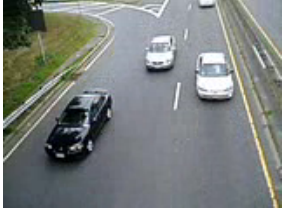

(b)
Fig. 9: Three car sequence.

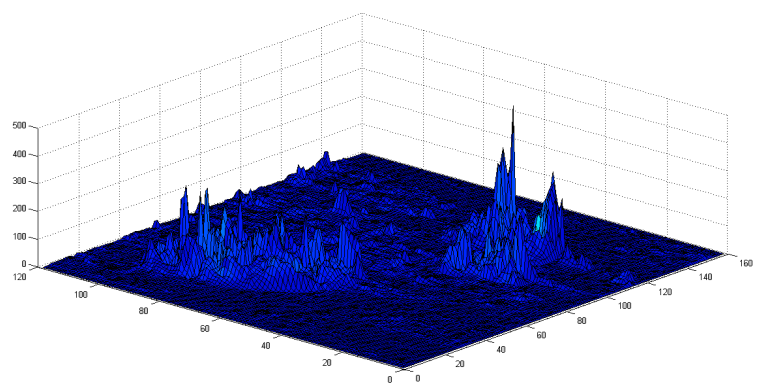

Fig. 10: 3 - D Motion histogram for three car sequence.

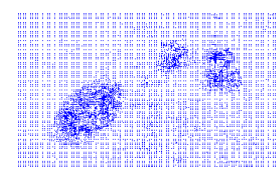

(a)

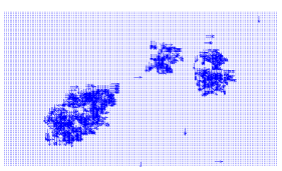

(b)

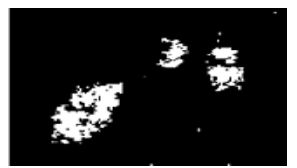

(c)

Fig. 11: (a) Motion vectors. (b) Refined motion vectors. (c) Refined motion mask.

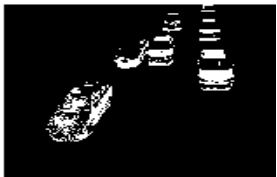

(a)

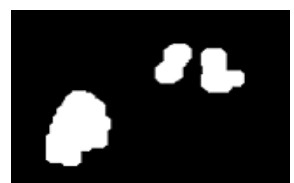

(c)

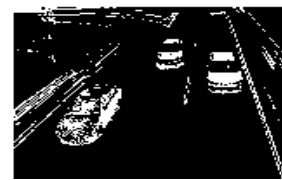

(b)

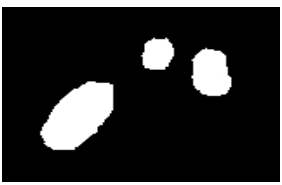

(d)
Fig. 12: (a) Result of frame difference method. (b) Result of background subtraction method. (c) Result of proposed method. (d) Ground truth.

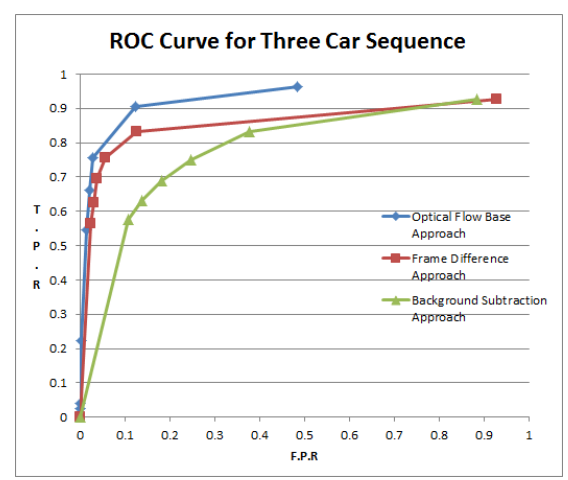

Fig. 13: ROC Curve for three car sequence. 


\subsection{Highway Sequence (Test Sequence -3)}

Figure 14 shows original highway sequence. Figure 15 represent its motion historgram. Result of optical flow algorithm as motion vectors followed by refined motion vectors as an outcome of thresholding function and finally refined motion masks are presented in Figure 16. Figure 17 displays result of frame difference, background subtraction and the result of proposed method and ground truth. In the "Highway traffic sequence" (see figure (14), there emerges small boundaries of stationary objects in the results of background subtraction. Result of frame difference shows that there exists false car detection with some of the true car detection. Using suggested technique, we can achieve much improved end result, even if there is lots of small dynamic movements due to camera vibration, changes in intensity of natural light and small dispersive movements of trees and vehicles at a far distance of the highway. Figure 18. shows result comparison on ROC Curve. As a conclusion from above discussion and figures, it is evident that proposed method gives much better results than the frame difference and the background subtraction.

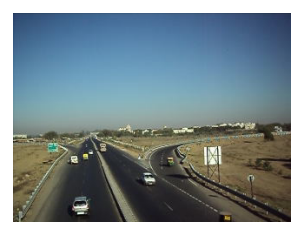

(a)

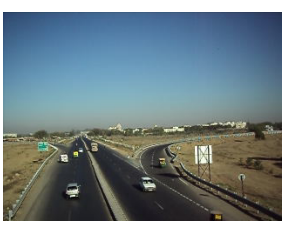

(b)
Fig. 14: Highway traffic sequence.

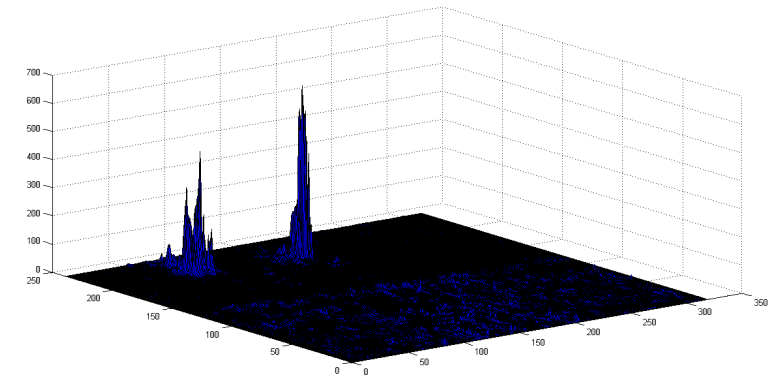

Fig. 15: 3 - D Motion histogram for highway traffic sequence.

\section{QUANTITATIVE EVALUATIONS (ROC) AND COMPUTATIONAL COST ANALYSIS}

The evaluation of suggested method was carried out quantitatively on the selected sequences using receiver operating characteristic curve (ROC). The ROC curve was plotted by taking all probable threshold values, and for each value, plotting of sensitivity value on the y-axis against the (1- specificity) value on the $\mathrm{x}$-axis carried out.[10] As threshold is the main controlling parameter for the suggested method. We have used many different value of threshold to generate the ROC plot. Similarly, ROC curve of the frame difference and the background subtraction method are plotted for

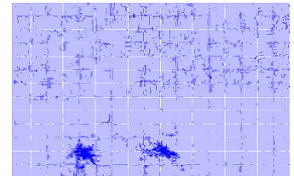

(a)

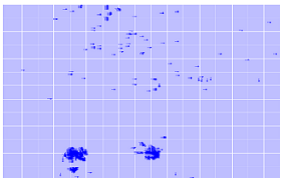

(b)

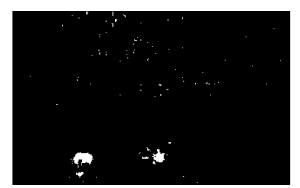

(c)

Fig. 16: (a) Motion vectors. (b) Refined motion vectors. (c) Refined motion mask.

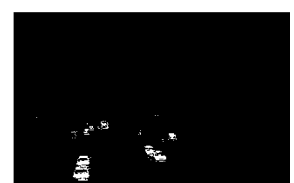

(a)

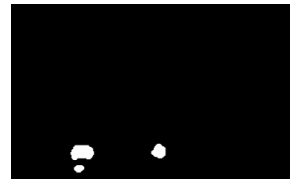

(c)

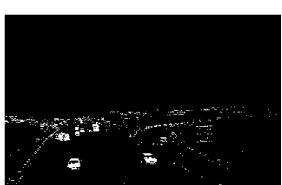

(b)

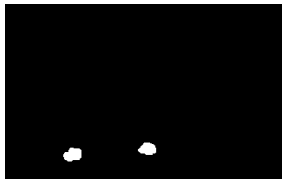

(d)
Fig. 17: (a)Result of frame difference method. (b) Result of background subtraction method. (c)Result of proposed method. (d) Ground truth

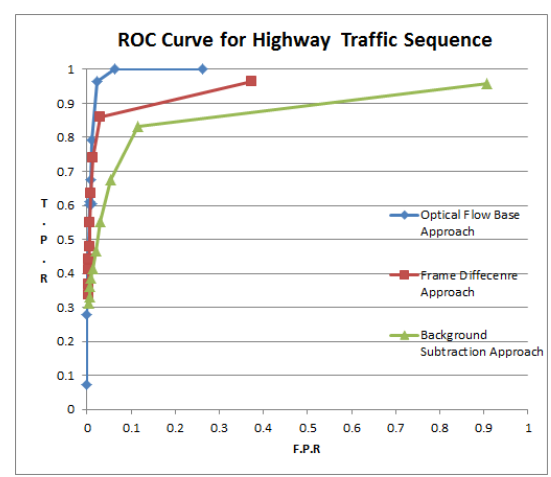

Fig. 18: ROC Curve for highway traffic sequence. 
comparison. The resultant ROCs are given in figures 813 and 18. It shows that the performance of the suggested method improves significantly than the frame difference and the background subtraction method in all three sequences. Furthermore, in "Black Car Sequence" and "Highway Traffic Sequence", the results of proposed method are very close to those of the optimal classifier. Morphological operation helps significantly in improving the result by shaping object.

Computational cost analysis First image sequence was sampled to $120 \times 160$ at frame rate 15 frames/s and second image sequence was sampled to $240 \times 320$ and at a frame rate 29 frames/s. CPU time consumed for each frame is $0.11 \mathrm{~s}, 0.12 \mathrm{~s}, 5.41 \mathrm{~s}$ and $0.11 \mathrm{~s}$, $0.12 \mathrm{~s}, 10.35 \mathrm{~s}$ for frame difference method, background subtraction method and optical flow method respectively. Result shows that time for our method is more than traditional methods due to calculation of motion vector for each pixel which are in motion. Time of computation of in search region has huge bearing on search region and it should be take in consideration.

\section{CONCLUSION}

Method using an optical flow and motion histogram techniques has been discussed in this paper. Optical flow was used to identify movement of pixel and motion histogram was used to differentiate dynamic background and foreground. Using morphological operation aperture effect of optical flow was reduced and shape of object was obtained. This method is found to be superior than frame difference and background subtraction method. Higher computational time is only the disadvantage. In future modifications in the algorithm is required for reduction in computational time. Method will be used to test different condition scene such that raining, low light condition, indoor video and this results will be compared with other standard methods.

\section{Acknowledgment}

We are very much thankful to Dr. Amit Ganatara, Dean, Charusat university for providing opportunity for research. We also take opportunity to thanks Dr. R.A.Thakkar for his support and guidance for this topic.

\section{REFERENCES}

[1] Soon Yong Park., Chang Joon Park, and Inho Lee, "Moving Object Removal and Background Completion in a Video Sequence",citeseerx.ist.psu.edu,doi:10.1.1.118.385,2008

[2] Shao-Yi Chien.,Shyh-Yih Ma, and Liang-Gee Chen, "Efficient Moving Object Segmentation Algorithm using Background Registration Technique",IEEE transcation on circuit and system of video technology, 12 (7), July-2002,pp.577586.

[3] Jie Cao, Li Li, "Vehicle Objects Detection of Video Image Based on Gray Scale Characteristics", in First International workshop on Education technology and computer science, March-2009, pp.936-940.

[4] Liu Liu., Jian-Xun Li., "A Novel Shot Segmentation Algorithm based on Motion Edge feature", in Symposium on Photonics and Opto electronic,19-21 June,2010, pp.1-5.

[5] Jie he,Kebin Jia, Zhuoyi Lv,'A Fast and Robust Algorithm of Detection and Segmentation for Moving Object", in Fifth International Conference on Intelligent Information and Multimedia Signal Processing, 12-14 Sept.2009, pp.718-721.
[6] Shijie Zhang,Fred Stentiform., "Motion Segmentation using Region Growing And An Attention Based Algorithm", in Fourth European Conference on Visual Media Production, 27-18,Nov.-2007, pp.1-8.

[7] Saeed Kamel,Hossein Ebrahimnezhad, Afshin Ebrahimi.,"Moving Object Removal in Video Sequence and Background Restoration using Kalman Filter", in International Symposium on Telecommunications, 27-28 Aug-2008, pp.580-585.

[8] Wei Zhang, Q.M. Jonathan Wu, Hai bing Yin, "Moving vehicles detection based on adaptive motion histogram",ElsevierDigital Signal Processing 20 ,2010,pp793-805.

[9] B.D. Lucas and T. Kanade, "An Iterative Image Registration Technique with an Application to Stereo Vision", in DARPA Image Understanding Workshop", 1981, IJCAI, pp121-130.

[10] "Receiver Operating Characteristics (ROC) Curve Preparation - A Tutorial",'Boston: Massachusetts Water Resources Authority Report" ENQUAD 2005.

[11] B.K.P. Horn and B.G. Schunck, "Determining Optical Flow", Artificial Intelligence",17, 1981,pp 185-204.

[12] E.P. Simoncelli, "Design of multi-dimensional derivative filters", IEEE International Conference on Image Processing", Vol.1,1994,pp790-793.

[13] Anand Paul, K. Bharanitharan and Jiaji Wu, "Algorithm and Architecture for Adaptive Motion Estimation in Video Processing", IETE Technical Review, Vol. 30, Issue 1,Jan-Feb2013, pp 24-30.

[14] J.L.Barron and N.A.Thacker "Tutorial: Computing 2D and 3D Optical Flow", Tina Memo No. 2004-012 